\title{
Design of UAV Airborne Reconnaissance and Rescue Based on
}

\section{ATMEGA2560}

\author{
Wen Yao Liu ${ }^{1, a^{*}}$, Cheng Zhan Peng ${ }^{2, b}$, Feng Wang ${ }^{3, c}$, Wei Jian $\mathrm{Mo}^{4, \mathrm{~d}}$ and Zhi \\ Ping Wan ${ }^{5, \mathrm{e}}$
}

\author{
$1,2,3,4$, 5Xinhua College of Sun Yat-sen University, Dongguan 523133, China \\ a506250345@qq.com, b871114246@qq.com, ${ }^{\mathrm{c}} 78133090 @ q q . c o m,{ }^{\mathrm{d}} 472252720 @ q q . c o m$, \\ e7620674@qq.com,
}

Keywords: Four-rotor; UAV; Wireless; Global positioning; Rescue.

\begin{abstract}
To solve the problem of accident rescue field, the paper designed an aerial rescue investigation drones based on ATMEGA2560.Using GPS global positioning system, PID control algorithm and ATEMGA2560 chip as the core controller. Hardware system includes: core control module based on ATMEGA2560, video surveillance module, global positioning (GPS) module, ultrasonic ranging module, human infrared sensor module, a wireless remote control module, wireless data transmission module. Development and debugging again on open source code by Arduino development environment, we achieve the UAV remote control, search and rescue proven road, designated search and rescue, remote wireless video real-time monitoring, environmental monitoring of other functions. Thus, on the condition of the accidents, the UAV can improve the quality of rescuing, reduce the risk of rescuers and provide a variety of favorable conditions for searching and rescuing on the field which can make rescue work more efficiently.
\end{abstract}

\section{Introduction}

With the advancement of technology and improvement of people's living standards, people enjoy life performance increasingly apparently. However, the Probability of accident began to rise. Due to communications infrastructure damage and difficult to establish contact with the trapped workers in these accident rescue work, so we designed a UAV quick search and rescue in the wild. Aerial rescue investigation drones is an effective tool to solve this problem. UAV based on ATMEGA2560 controller chip, It is made up of gesture detection module, global positioning (GPS) module, ultrasonic ranging module, human infrared sensor module, wireless remote control module, wireless data transmission module and other components. In the rescue work, the UAV can be real-time operated by rescue workers.

\section{UAV Functional Architecture}

The design of UAV airborne reconnaissance and rescue based on ATMEGA2560 mainly realize four functions: real-time video monitoring, sentinel searching, rescue environment detecting and autonomous cruise searching, and one of the most critical point is the design of sentinel searching. Meanwhile, introducing the PID control algorithm to make the UAV can stably fly in the search and rescue operations and to improve the stability and reliability of the UAV system functions. 
Real-time video monitoring. In many accident rescues, for example, travel enthusiasts climbing missing, tourist coaches rushed into the canyon, travel enthusiast fell off a cliff. And often due to dangerous environment and unknown accident site status cause rescuers difficult to enter the scene to rescue. In order to improve the efficiency of search and rescue, UAV use of wireless image transmission technology, image processing technology, as well as the PC real-time monitoring capabilities to get the latest information of the wounded and the environment.

The sentinel search function. In the rescue work, because the location is difficult to determine and result in affecting the rescue work. This design uses the Global Positioning System (GPS) module to obtain accurate position information and sends location information via wireless data transmission module to the host computer. Meanwhile, achieve PC real-time plan rescue line and designate cruise search and rescue capabilities, greatly reduces the intensity of the search.

The rescue environment detecting function. In rescue work, if the environment of the accident scene was unknown, lives of rescue workers and the wounded would be threatened. We will add the infrared human body detection module in the UAV design, and it is convenient to search and rescue the wounded. Based on various factors mentioned above, some appropriate detection modules will be added to the design to make the UAV can detect environmental conditions information in the search and rescue work to facilitate rescue the wounded, and guarantee lives of aid workers to be safe.

The autonomous cruise search and rescue function. Autonomous cruise function is the interaction between host computer and a wireless controller, through the Mission planner software programming automatic cruise line, designated location search. And sends the information of corresponding planning to the UAV through the wireless data transmission module, then UAV executes the commands and realize autonomous cruise search function. Autonomous cruise search and rescue capabilities are designed for increasing the air search and rescue efforts; and at the same time, reducing the output of human rescuers.

\section{The hardware circuit design and implementation}

In this design we use the ATMEGA2560 chip as the core, including core control module based on ATMEGA2560, video monitoring module, global positioning (GPS) function module and so on.

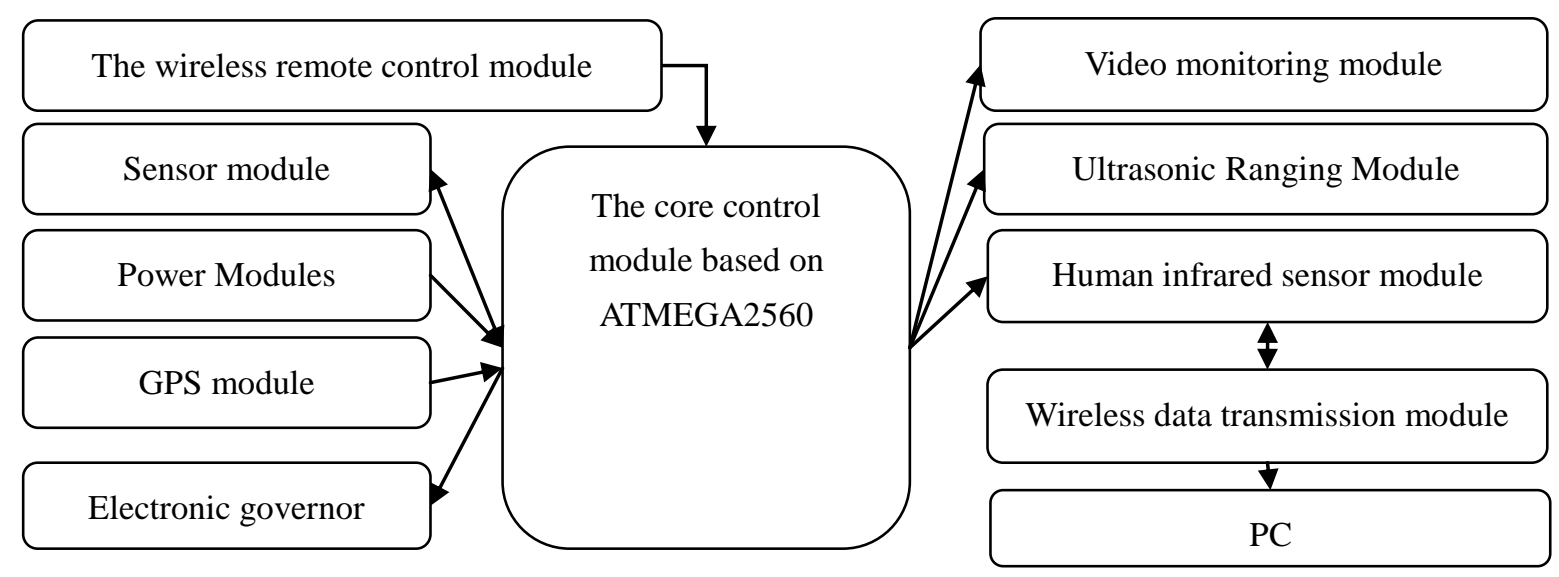

Figure. 1 The hardware structure chart

The core control module. The core control module is composed of ATMEGA2560 main control chip, ATMEGA168 PPM decoder chip, MPU6050 three axis accelerometer, three axis gyro, ADS7844 chip and so on. By using MPU6050 three-axis accelerometer, three-axis gyroscope to detect UAV attitude, and to control UAV through two levels of PID control mode of flight .The Triaxial accelerometer is used to measure linear acceleration of UAV. Its output value and the tilt 
angle is nonlinear, and increases as the tilt angle changes appear as a sinusoidal function. The first level of PID control method is navigate stage, second level is control stage. Navigation class computing focused medium_loop () and fast_loop () of update_curren_flight_mode () function, the control stage focus fast_loop () of stabilize () function. Navigation PID control solved is how UAV setting airspeed at a predetermined height and flying to the target. Fig. 2 is a schematic of MPU 6050 .

By the PID algorithm to calculate the desired pitch angle, throttle and roll angle, and then handed over to the control level to control solver. According to the pitch angle control level required, throttle and roll angle, combined with the current UAV attitude solution calculates the appropriate amount of steering control, so that UAV maintain a predetermined pitch angle, roll angle and direction angle. By the installation in four independent brushless motor of rigid cross structure to drive four propellers generate upward thrust. Model diagram is shown in Fig. 3.

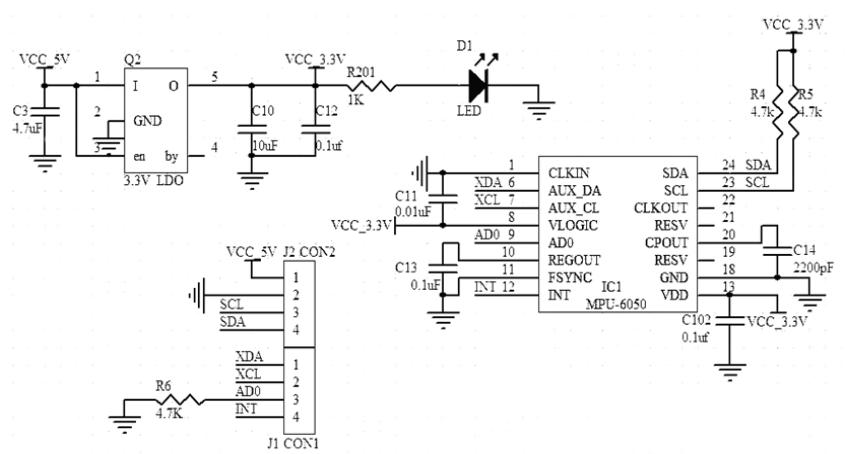

Figure. 2 The MPU 6050 schematics

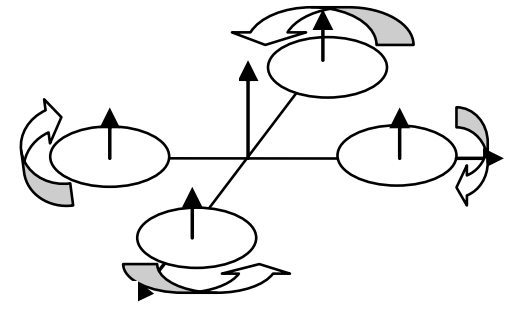

Figure. 3 The Dynamic Model

The video monitoring module. Video surveillance camera module consists of a wireless connection, and equipped with the latest image processing engine for the transmission of aerial reconnaissance collected image data, and then returned to PC. Because the module comes with compression algorithms, wireless data transmission greatly reduces the amount of data transmission.

\section{The Global Positioning (GPS) module.}

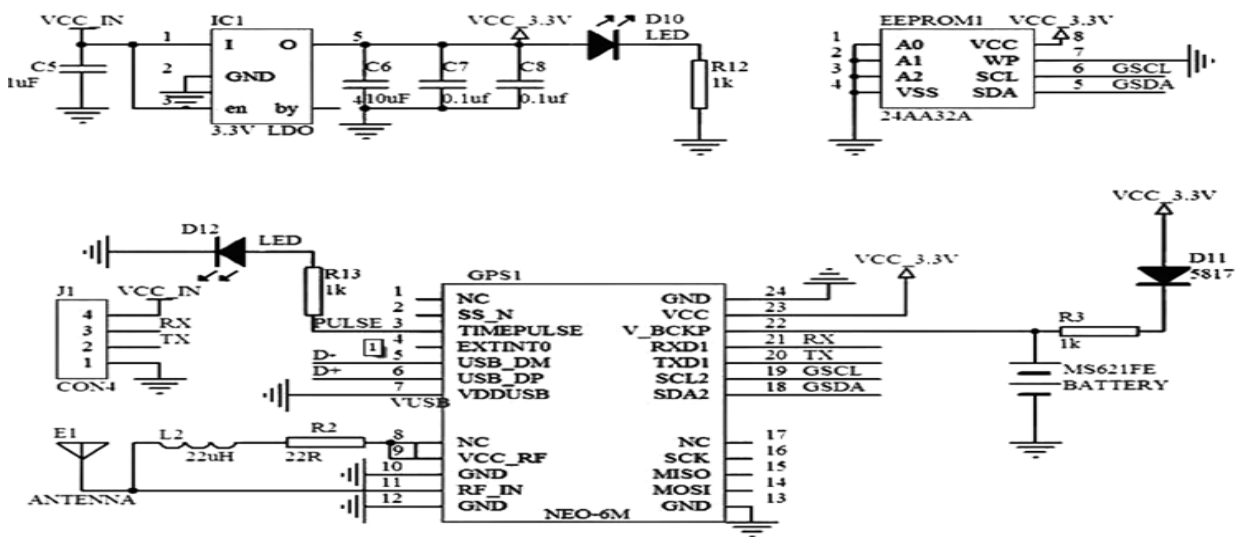

Figure. 4 The UBLOX NEO-6M GPS Schematics

We use the global positioning module called UBLOX NEO-6M GPS, and it is a high performance GPS positioning module. The module consists of UBLOX NEO-6M module, and it comes with a high-performance passive ceramic antenna the module tracking sensitivity is $-162 \mathrm{dBm}$, cold start sensitivity of $-148 \mathrm{dBm}$, configurable power management, GPS / SBAS hybrid 
engine (WAAS, EGNOS, MSAS) and the use of anti-jamming technology to support AssistNow Online and AssistNow Offline and so on. The Circuit diagram is shown in Fig. 4.

\section{The ultrasonic ranging module and human infrared sensor module}

The ultrasonic ranging module. The system made up of ultrasonic ranging module HC-SR04,it can detect the range of $2 \mathrm{~cm} \sim 450 \mathrm{~cm}$, accuracy up to $0.3 \mathrm{~cm}$. Using $\mathrm{I} 2 \mathrm{C}$ serial interface to communicate with the core control module, automatic response $\mathrm{I} 2 \mathrm{C}$ serial control commands. Using IO trigger measure distance, the automatic transmission Eight $40 \mathrm{kHz}$ square wave, and detects whether a signal is returned. Using ultrasonic ranging module, makes the rescue efforts in the air UAV avoid obstacles, and it can also be used to measure the approximate distance UAV and wounded.

The human infrared sensor module. Pyroelectric infrared sensor will return electrical signal if it detects a human or animal emit infrared. Human infrared sensor module pyroelectric infrared sensor This design uses infrared rays(about 10um) on the human body emitted a constant temperature made pyroelectric effect. Pyroelectric effect is similar with the piezoelectric effect, and it is due to temperature changes caused by the phenomenon of crystal surface charge. Charge $\Delta \mathrm{Q}$ pyroelectric effect produced will disappear with the combination of ions in the air, namely when the ambient temperature is stable and unchanging, $\Delta \mathrm{T}=0$, then the sensor is no output. When the body enters the detection zone, the temperature and ambient temperature is difference, resulting in $\Delta \mathrm{T}$ output.

\section{The wireless remote control module, wireless data transmission module}

The wireless remote control module. In order to control UAV by wireless remote control, with uses a WFR07S 2.4GHz receiver control module to connected to the core control module, enabling wireless remote communication with the remote control terminal UAV. By remote control terminal to send control commands to WFR07S $2.4 \mathrm{GHz}$ receiver, its control commands via the communication protocol control commands sent to ATMEGA2560 core control module.

The wireless data transmission module. Wireless data transmission module module uses digital signal processing, digital modulation and demodulation, with forward error correction, balanced sof $\mathrm{t}$ decision functions such CUAV Radio 3DR data transmission module. CUAV Radio 3DR transmiss ion module using two-way communication protocol of MAVLink framework, part of the protocol c ode is as follows:

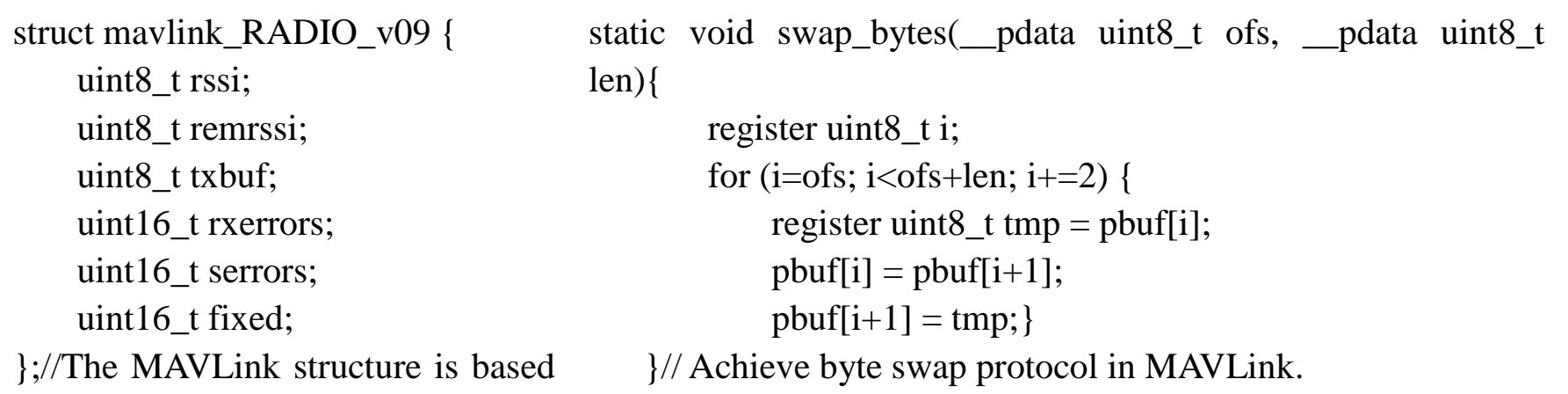
define on the above information 


\section{The Four-rotor UAV flight}

Software simulation of flight and Outdoor real flight.Software simulation flight and Outdoor real flight are shown in Fig. 5.

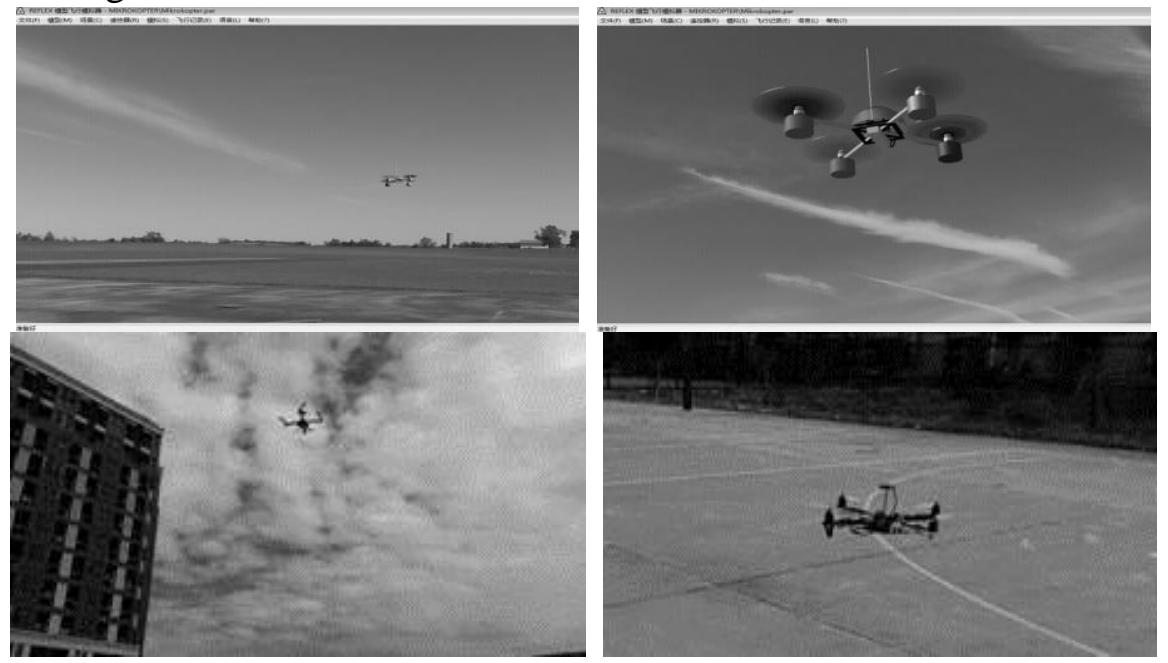

Figure. 5 The Four-rotor UAV flight image

\section{Summary}

In many fields, such as civil, rescue, military and so on, four-rotor UAV plays an increasingly significant role. Aerial rescue investigation drones based on ATMEGA2560 can establish contact with the trapped workers and survey the disaster. The design of the UAV is essentially a self-positioning and automatic flying four-rotor aircraft. The camera of UAV transports back rescue information by real-time image transmission module, and it suitable for disaster survey.

\section{References}

[1] Wang ZH, Huang XN, Liang K, Li SB, Yang Z. Study of Transmission Line Inspection System Based on Four-Rotor UAV [J]. China Power,2012,45(10):59-62.

[2] Wang SH, Yang Y. Study of Four-Rotor Aircraft Pose Estimation And Control Based on Kalman Filter. [J]. Control Theory \& Applications,2013,30(9):1109-1115.

[3] Zhao CJ, Gong X, Bai Y, Xu ZJ, Xu DF, Gao QJ. The Interference And Suppression of Lift Fluctuations in Four-Rotor [J]. Optical Precision Engineering,2014,22(09):2431-2437.

[4] Li JY, Zhang TM, Peng XD, Ma Xuedong, Ke CC, Zeng XS. Four-Rotor in Farmland Position Information Collection Platform Design And Experiment. [J]. The Learned Journal of Agricultural Machinery,2013,44(05):202-206+212.

[5] Yang HL, Jiang B, Zhang K. Four-Rotor Helicopter Attitude System of Self-healing Control Directly [J]. Control Theory And Applications,2014,31(08):1053-1060.

[6] Jiang J, Qi JT, Han JD. Facing The Maneuvering Flight of Rotor Aircraft Design And Modeling And Control [J]. Science Bulletin,2013,58(Supplement II ):135-144.

[7] Chen YM, He YL, Kong LB, Zhou MF. Four-Rotor Decentralized PID Neural Network Control[J]. The Learned Journal of Chinese Inertial Technology ,2014,(02):185-190.

[8] Chen BQ, Yang YM, Zheng LH, Wen HT. The Automatic Registration of Four-Rotor UAV Image Based on SIFT And TPS Algorithm [J]. Sensing Technology And Application,2013,28(04):604-609.

[9] Zhan L, He RQ, Xie Y,LONG Y. The System of Intelligent Navigation Based on Micro Four-Rotor UAV[J]. Electronic Measurement Technology ,2011, 34(06):1-3+29. 
[10]Chen HB, Shu GH. The Design of The Four-Rotor Aircraft [J]. Laboratory Research And Exploration,2013,32(03):41-44. 